\title{
GENERALIZED CONTINUITY EQUATION FOR QUASI-HERMITIAN HAMILTONIANS
}

\author{
Amine B. Hammou* \\ USTO-Mohamed Boudiaf, Département de Physique, BP 1505 El M'naouer, Oran, Algeria \\ * corresponding author: hbamine@gmail.com
}

Abstract. The continuity relation is generalized to quasi-Hermitian one-dimensional Hamiltonians. As an application we show that the reflection and transmission coefficients computed with the generalized current obey the conventional unitarity relation for the continuous double delta function potential.

KEYWORDS: complex scattering potential, metric operator, quasi-Hermitian, $\mathcal{P} \mathcal{T}$-symmetry.

\section{INTRODUCTION}

In [1, scattering from a discrete quasi-Hermitian delta function potential was studied and a generalized continuity relation was obtained in the physical Hilbert space $\mathcal{H}_{\text {phys }}$. A generalized probability current density was defined. Using a quasi-Hermitian toy model of discrete delta function potential, it was shown that the reflection and transmission coefficients computed using this current obey the unitarity relation.

In this paper we will review the construction of the generalized continuity relation of [1] and then apply it to the continuous double delta function potential where a metric was obtained to first order in the imaginary part of the potential strength [2]. We will then compute the reflection and transmission coefficients in the physical Hilbert space $\mathcal{H}_{\text {phys }}$ and show that they indeed obey the unitarity relation. This result comes as a surprise since, although the potential is local, the metric is highly non local and so the existence of asymptotic states is questionable [3 5]. This is as far as I know the first example of a quasi-Hermitian Hamiltonian with a non-diagonal metric that possesses a conserved probability current density. Recently it has been found [6] that, for the non-Hermitian complex PT-symmetric version of the Scarf II potential, unitarity is obeyed for a particular choice of the parameters.

\section{The Continuity Relation in $\mathcal{H}_{\text {phys }}$} A Hamiltonian $H$ that obeys

$$
H^{\dagger}=\eta H \eta^{-1}
$$

is said to be quasi-Hermitian [7]. Where $\eta$ is a positive definite metric operator. By an appropriate modification of the inner product on the Hilbert space, quasi-Hermitian operators can be made Hermitian 8]. The inner product of the new Hilbert space is given by $\langle\cdot \mid \cdot\rangle_{\eta}:=\langle\cdot \mid \eta \cdot\rangle$, where $\langle\cdot \mid \cdot\rangle$ is the inner product which defines the original Hilbert space $\mathcal{H}$. The new Hilbert space endowed with the inner product $\langle. \mid .\rangle_{\eta}$ is recognized as the physical Hilbert space $\mathcal{H}_{\text {phys }}$ in which $H$ acts as a Hermitian operator [8]. A generalized continuity equation in the physical Hilbert space $\mathcal{H}_{\text {phys }}$ was defined in [1] as

$$
\frac{\partial}{\partial t} \rho_{\eta}(x)+\frac{\partial}{\partial x} j_{\eta}(x)=0
$$

in the $x$-representation, where

$$
\rho_{\eta}(x)=\int d y \psi^{*}(y) \eta(y, x) \psi(x)
$$

is the probability density. The probability current density is given by

$$
\begin{aligned}
j_{\eta}(x)=i \int d y\left(\psi^{*}(y) \eta(y, x) \frac{\partial \psi(x)}{\partial x}\right. & \\
- & \left.\frac{\partial\left(\psi^{*}(y) \eta(y, x)\right)}{\partial x} \psi(x)\right) .
\end{aligned}
$$

\section{The Continuous double Delta FUNCTION POTENTIAL}

In this section, we will test the continuity relation obtained from the generalized current 2.4 in the continuous case. There are very few cases in the literature where the metric was computed [2, 9-11]. Let us consider the double delta function potential given by

$$
V(x)=i \lambda(\delta(x+a)-\delta(x-a))
$$

with the scattering wave function, in the situation where the plane wave comes in from the left and is either reflected or transmitted at the delta function potential, is of the form

$$
\begin{cases}\psi_{<}(x)=e^{i k x}+r e^{-i k x} & \text { if } x \ll-a, \\ \psi_{>}(x)=t e^{i k x} & \text { if } x \gg a,\end{cases}
$$

where $\psi_{<}(x)$ and $\psi_{>}(x)$ are respectively the incoming and the outgoing wave functions. The metric was given in 2] as a perturbative series in $\lambda$, to first order it is given by

$$
\eta(x, y)=\delta(x-y)+\eta^{(1)}(x, y)+O\left(\lambda^{2}\right)
$$


where

$$
\begin{aligned}
& \eta^{(1)}(x, y)=i \frac{\lambda}{2} \operatorname{sign}(x-y)(\theta(x+y+2 a) \\
&-\theta(x+y-2 a)) .
\end{aligned}
$$

Let us apply our formula for the generalized continuity relation introduced in 2.4 to this case. Therefore we should compute the functions

$$
\chi(x)=\psi(x)+\int \eta^{(1)}(x, y) \psi(y) d y .
$$

A close inspection of the metric shows that for both $x \ll-a$ and $x \gg a$ with $-(x+2 a)<y<-(x-2 a)$ we have $\eta^{(1)}(x, y) \neq 0$; otherwise it is zero.

We therefore obtain for $x \ll-a$

$$
\begin{aligned}
\chi_{<}(x)=\psi_{<} & (x)-i \frac{\lambda}{2} \int_{-(x+2 a)}^{-(x-2 a)} \psi_{<}(y) d y \\
= & e^{i k x}+\left(r-i \frac{\lambda}{k} t \sin 2 a k\right) e^{-i k x}
\end{aligned}
$$

and for $x \gg a$ we find

$$
\begin{aligned}
& \chi_{>}(x)=\psi_{>}(x)+i \frac{\lambda}{2} \int_{-(x+2 a)}^{-(x-2 a)} \psi_{<}(y) d y \\
& =\left(t+i \frac{\lambda}{k} r \sin 2 a k\right) e^{i k x}+i \frac{\lambda}{k} \sin 2 a k e^{-i k x} .
\end{aligned}
$$

The second term in this function can be interpreted as a reflected wave function traveling from right to left and therefore it has no physical meaning [4, 5]. This causes no problem in our approach since $\chi(x)$ has no physical interpretation. The generalized reflection coefficient is easily computed using its definition in terms of reflected and incident generalized currents that can be worked out using (2.4), and is given by

$$
\mathcal{R}=|r|^{2}+\frac{i \lambda}{4} t^{*} r \sin 2 a k .
$$

Similarly the generalized transmission coefficient can be computed using the transmitted and incident currents, and is given by

$$
\mathcal{T}=|t|^{2}-\frac{i \lambda}{4} r^{*} t \sin 2 a k
$$

leading to

$$
\mathcal{R}+\mathcal{T}=|r|^{2}+|t|^{2}+\frac{i \lambda}{4}\left(t^{*} r-r^{*} t\right) \sin 2 a k
$$

Note that if we use the complex conjugate of relation (2.4) to compute the generalized reflection and transmission coefficient we obtain the same result. This is easily seen from the fact that the reflection and transmission coefficients are real and hence the currents are real too. The first term in both $\mathcal{R}$ and $\mathcal{T}$ are obviously real and the second terms are also real because of the relation $t^{*} r=-r^{*} t$, which is a general feature of $\mathcal{P} \mathcal{T}$-symmetric models $[12$.
For the model at hand (3.1), the reflection and transmission amplitudes can be easily worked out, leading to

$$
r=i t \frac{\lambda}{k}\left(1-\frac{\lambda}{2 k}\right) \sin 2 a k
$$

and

$$
t=\frac{1}{1+i \frac{\lambda^{2}}{2 k^{2}} e^{2 i a k} \sin 2 a k} .
$$

It is worth noting that if we do usual quantum mechanical scattering $(\eta(x, y)=\delta(x, y))$ by using the PT-symmetric double delta function potential we will not have unitarity

$$
|r|^{2}+|t|^{2}=\frac{1+\frac{\lambda^{2}}{k^{2}}\left(1-\frac{\lambda}{2 k}\right)^{2} \sin ^{2} 2 a k}{1-\frac{\lambda^{2}}{k^{2}}\left(1-\frac{\lambda^{2}}{4 k^{2}}\right) \sin ^{2} 2 a k} .
$$

By using the expressions 3.11 and 3.12 we obtain

$$
t^{*} r=-r^{*} t=i|t|^{2} \frac{\lambda}{k}\left(1-\frac{\lambda}{2 k}\right) \sin 2 a k
$$

and after a straightforward computation we can show that

$$
\mathcal{R}+\mathcal{T}=1
$$

We have obtained a unitarity relation in the Hilbert space $\mathcal{H}_{\text {phys }}$. Note that this result is exact in $\lambda$, as we have used the full expressions of the transmission (3.12) and reflection amplitudes (3.11) in terms of $\lambda$ (to all orders). This is a puzzling result that we have yet to understand. It is clear that if one uses the expressions for $t$ and $r$ to first order in $\lambda$, the relation (3.15 holds trivially. A possible explanation is that the higher orders in $\lambda$ of the metric somehow will not change the result of the continuity relation. For this to be true, the higher order corrections should not contribute to the functions $\chi_{<}(x)$ and $\chi_{>}(x)$.

\section{Conclusion}

We have been able to show that the generalized current defined with respect to the metric $\eta$ is conserved

$$
\left(J_{\eta}\right)_{\text {in }}-\left(J_{\eta}\right)_{\text {out }}=0
$$

and the corresponding generalized reflection and transmission coefficients obey the unitarity relation for the quasi-Hermitian continuous double delta function potential.

Although the metric used in the continuous case is perturbative in the strength of the imaginary part of the potential $\lambda$, the results of the unitarity relation were obtained using the expression for the amplitudes to all orders in $\lambda$. And so this arises a question that deserves further investigation. To this end, one has to work out the metric to a higher order in $\lambda$. Finally an interesting and urgent question is to understand the physical implementation of the metric $\eta$ in these scattering processes. 


\section{ACKNOWLEDGEMENTS}

I would like to thank the Abdus Salam International Center for Theoretical Physics ICTP, Trieste, Italy for Hospitality and support. I would also like to thank M. Znojil for helpful discussions and for organizing the conference "Analytic and Algebraic methods in Physics X", Prague.

\section{REFERENCES}

[1] Amine B. Hammou, J. Phys. A: Math. Theor. 45 (2012) 215310.

[2] H. Mehri-Dehnavi, A. Mostafazadeh, A. Batal, J. Phys. A43, 145301 (2010).

[3] M. Znojil, Phys. Rev. D78, 025026 (2008).

[4] H. F. Jones, Phys. Rev. D76, 125003 (2007).
[5] H. F. Jones, Phys. Rev. D78, 065032 (2008).

[6] Z. Ahmed, arXiv:1207.6896

[7] F. G. Scholtz, H. B. Geyer and F. J. W. Hahne, Ann. Phys. 213, 74 (1992).

[8] A, Mostafazadeh, Int. J. Geom. Meth. Mod. Phys. 7, 1191 (2010).

[9] A. Mostafazadeh, J. Math. Phys. 46, 102108, (2005).

[10] A. Mostafazadeh, J. Phys. A: Math. Gen. 39, 13495 (2006).

[11] A. Mostafazadeh, J. Phys. A39, 10171 (2006).

[12] F. Cannata, J.-P. Dedonder and A. Ventura, Ann. Phys. 322, 397 (2007). 